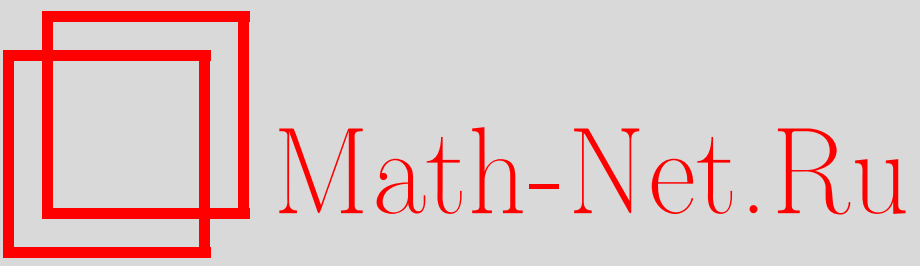

К. А. Макаров, В. В. Мележик, Две стороны медали: эффект Ефимова и коллапс в системе трех частиц с точечными взаимодействиями. I., ТМФ, 1996, том 107, номер 3, 415-432

DOI: https://doi.org/10.4213/tmf1166

Использование Общероссийского математического портала Math-Net.Ru подразумевает, что вы прочитали и согласны с пользовательским соглашением

http: //www . mathnet.ru/rus/agreement

Параметры загрузки :

IP : 107.22 .136 .117

26 апреля 2023 г., 10:44:22 
ТЕОРЕТИЧЕСКАЯ

И МАТЕМАТИЧЕСКАЯ

ФИЗИКА

Том 107, № 3

июнь, 1996

К. А. Макаров, В.В. Мележик

\section{ДВЕ СТОРОНЫ МЕДАЛИ: ЭФФЕКТ ЕФИМОВА И КОЛЛАПС В СИСТЕМЕ ТРЕХ ЧАСТИЦ С ТОЧЕЧНЫМИ ВЗАИМОДЕЙСТВИЯМИ. І.}

Рассматриваются системы трех частиц с точечными взаимодействиями с внутренней структурой. Проведена полная классификация систем, и указаны условия при которых соответствующий оператор энергии полуограничен снизу.

Мы посвящаем настояшую работу памяти Станислава Петровича Меркурьева, которого вот уже три года как нет среди нас. Нет и семинаров, проходивших по четвергам в одной из аудиторий главного здания университета, на которых С.П.Меркурьев, выслушав докладчика или порой только начав его слушать, уже видел проблему изнутри, предлагая возможные более экономные варианты ее решения, или, напротив, выражал свое критическое отношение к предмету изложения, сохраняя при этом удивительную толерантность. Готовя рукопись данной работы, мы вспоминали перефразированное высказывание П.Р.Халмоша: "Сперва выбери потенциального читателя (слушателя), а потом пиши (говори).” Следуя этому совету, мы сделали свой выбор в пользу С.П.Меркурьева, надеясь воспроизвести традиционньй стиль заседаний его семинара, как бы приглашая читателя стать участником одного из них, где Станислав Петрович незримо присутствует.

\section{1. ВВЕДЕНИЕ}

60-е годы ознаменовались двумя примечательными событиями в спектральной теории многочастичного уравнения Шредингера. Первое из них - обнаружение коллапса в системе трех частиц с взаимодействиями типа $\delta$-функции $[1,2]$, второе - эффект Ефимова - наличие бесконечного дискретного спектра в некоторых системах трех частиц с быстроубываюшими потенциалами [3].

Исторически первое математическое объяснение эффекта Ефимова принадлежит Фаддееву, который заметил ${ }^{1)}$, что доказательство существования бесконечной серии связанных состояний оператора энергии системы трех частищ с быстроубывающими потенциалами взаимодействия (при наличии виртуальных уровней в двухчастичных подсистемах) сводится к изучению условий разрешимости при больших отрицательных энергиях уравнений Скорнякова-Тер-Мартиросяна [4], уточненных Даниловым [5]. Исследование условий разрешимости было проведено Фаддеевым и Минлосом $[1,2]$. Фад-

\footnotetext{
1) Частное сообщение Л.Д.Фаддеева.
} 
деев обратил внимание Яфаева на эту задачу, в результате чего появилась полная математическая теория эффекта Ефимова [6], носящая, правда, качественный характер.

Явные указания на тесную количественную связь между асимптотикой собственных значений, у ходящих на минус бесконечность, для системы трех частиц с $\delta$-потенциалами и асимптотикой аккумуляции собственных значений к порогу развала в эффекте Ефимова содержатся в работе [7]. В этой работе выдвинута гипотеза об универсальности эффекта Ефимова, состоящая в том, что указанная асимптотика определяется лишь соотношением масс частиц и не зависит от деталей поведения двухчастичных потенциалов взаимодействия (при наличии виртуальных уровней в подсистемах, разумеется). Доказательство близкого (но более слабого) утверждения можно найти в работе [8].

Итак, коллапс в системе трех частиц с $\delta$-взаимодействиями и эффект Ефимова (по крайней мере в случае, когда в каждой двухчастичной подсистеме имеется виртуальный уровень) являются двумя сторонами одного и того же явления. Эта аналогия представляется, однако, неполной. Действительно, класс трехчастичных систем (с быстроубывающими взаимодействиями) можно разбить на 4 подкласса в соответствии с числом парных подсистем, в которых присутствует виртуальный уровень. При этом эффект Ефимова имеет место лишь только в двух из этих подклассов. Провести аналогичную классификацию трехчастичных систем с $\delta$-взаимодействиями так, чтобы в двух из таких подклассов оператор энергии был неполуограничен снизу, а в двух других явление коллапса отсутствовало бы, в принципе невозможно: любая самосопряженная реализация оператора энергии в таких системах является неполуограниченным снизу оператором [9]. Мы не имеем в виду, конечно, тривиальный случай, когда в одной (или нескольких) из подсистем частицы просто не взаимодействуют. Тем не менее в более широком классе систем с точечными взаимодействиями с внутренней структурой, предложенных и частично исследованных в работах [10-14], такая классификация все же возможна.

Первые примеры трехчастичных систем с точечными взаимодействиями, в которых явление коллапса не наблюдается, появились в работах Шондина [10] и Томаса [11].

После появления работы Павлова [15], где он предложил модель $\delta$-потенциала с внутренней структурой, рассматривая самосопряженные расширения оператора Лапласа с выходом из основного гильбертова пространства в произвольное гильбертово пространство, стало ясно, что класс $S$-матриц, отвечающих обычным $\delta$-потенциалам, существенно обогашается и параметризуется уже не вешественным параметром $\omega$ :

$$
S(k)=\frac{\omega-i k}{\omega+i k},
$$

как это имеет место в случае обычных $\delta$-взаимодействий, а произвольной $R$-функцией $\omega(z)$ параметра $z$

$$
S(k)=\frac{\omega\left(k^{2}\right)-i k}{\omega\left(k^{2}\right)+i k} .
$$

Отдавая дань истории, отметим, что первооткрывателем “внутренней” структуры следует считать Шрадера [16], который успешно использовал аналогичные расширения с выходом в одномерное пространство при регуляризации гамильтониана в нерелятивистской модели Ли.

В силу теоремы представления

$$
\omega(z)=A z+B+\int \frac{z s+1}{s-z} d \mu(s),
$$


где $A \geq 0, \operatorname{Im} B \geq 0$ и $\mu$-конечная борелевская мера, соответствуюшие модели естественно разделить на два класса в зависимости от того, содержит ли $R$-функция $\omega(z)$ в представлении Герглотца (1.3) “линейное слагаемое по $z$ " $(A>0)$ или нет $(A=0)$.

Мы выделим два случая (начиная с этого момента мы будем считать, что мера $\mu$ в представлении (1.3) имеет компактный носитель).

Случай а: $S$-матрица модели имеет высокоэнергетическое поведение, характерное для обычного потенциального рассеяния:

$$
S(k) \underset{k \rightarrow \infty}{\rightarrow} 1 .
$$

Случай б: $S$-матрица имеет “аномальное" поведение, характерное, в частности, для обычного $\delta$-потенциала:

$$
S(k) \underset{k \rightarrow \infty}{\longrightarrow}-1 .
$$

В [12] Павлов исследовал систему трех частиц с внутренней структурой таких, что в каждой из двухчастичных подсистем реализуется случай “а” и доказал полуограниченность трехчастичного оператора энергии системы. К сожалению, взаимодействия, предложенные в [12], оказались, строго говоря, не парными. Соответствующая модификация на случай парных взаимодействий содержится в работе одного из авторов [13], где дано прямое доказательство полуограниченности соответствуюшего гамильтониана через оценку его квадратичной формы. Теория рассеяния для таких систем развита в [14].

Случай трех частиц с $\delta$-потенциалами с внутренней “структурой”, когда хотя бы в одной из двухчастичных подсистем соответствующая $S$-матрица обладает аномальным высокоэнергетическим поведением, подробно не изучался.

В соответствии с классификацией (1.4), (1.5) мы приходим к естественному разбиению класса трехчастичных подсистем с точечными взаимодействиями на 4 подкласса. Эта классификация полностью аналогична классификации трехчастичных систем с быстроубывающими потенциалами взаимодействия с точностью до замены слов “в подсистеме $\alpha$ имеется виртуальный уровень" (резонанс при нулевой энергии) на выражение "в подсистеме $\alpha$ соответствуюшая $S$-матрица обладает аномальным поведением при больших энергиях". При этом справедливо следующее утверждение, слово в слово повторяющее формулировку эффекта Ефимова (см., например, [6-8]).

ТЕОрема 1.1. Всякая самосопряженная реализация оператора энергии системы трех частии с $\delta$-взачмодействием с внутренней структурой полуограничена снизу тогда и только тогда, когда по крайней мере две из трех $S$-матрии, отвечающих парным взаимодействиям, обладают нормальным высокоэнергетическим поведением, m. е.

$$
S_{\alpha}(k) \underset{k \rightarrow \infty}{\rightarrow} 1
$$

по крайней мере для двух $\alpha$ из множества $\alpha \in\{\{1,2\},\{2,3\},\{1,3\}\}$.

Часть этого утверждения в случае, когда все $S_{\alpha}(k) \rightarrow 1$ при $k \rightarrow \infty$, сформулирована впервые в работе Павлова [12] (см. также [13]). Идея классификации взаимодействия в терминах поведения соответствующих $S$-матриц двухчастичных подсистем при больших энергиях принадлежит Павлову [12].

В каждом из случаев мы опишем предгамильтонианы трехчастичных систем с точечными взаимодействиями как симметричные операторы в гильбертовом пространстве.

3 Теоретическая и математическая физика, т. 107, № 3, 1996 г. 
Мы докажем часть этой теоремы (см. лемму 4.1), относяшуюся к полуограниченному случаю: если по крайней мере в двух из двухчастичных подсистем $S$-матрицы обладают нормальным высокоэнергетическим поведением (1.4), то трехчастичный оператор энергии полуограничен.

Доказательство второй части импликации теоремы, касающейся неполуограниченности снизу оператора энергии (когда число двухчастичных подсистем с "аномальной” $S$-матрицей (1.5) не менее двух), следует оригинальному доказательству Фаддеева-Минлоса [2]. Необходимые для этого доказательства технические приемы можно извлечь, комбинируя результаты четырех работ: $[7,8,10,17]$, и мы оставляем для себя возможность опубликовать “детали” во второй части настоящей работы.

\section{2. ГАМИЛЬТОНИАН СИСТЕМЫ ТРЕХ ЧАСТИЦ С ТОЧЕЧНЫМИ ВЗАИМОДЕЙСТВИЯМИ КАК СИММЕТРИЧНЫЙ ОПЕРАТОР}

В настояшем разделе мы опишем оператор энергии системы трех частиц с внутренней структурой в импульсном представлении. Мотивировки $[12,13]$ и обсуждение парного характера рассматриваемых взаимодействий можно найти в $[13,14]$.

Преж де всего будем считать, что в пространстве $\mathbb{R}^{6}$ заданы три системы ортогональных координат (координат Якоби)

$$
P=k_{\alpha} \oplus p_{\alpha}, \quad \alpha=1,2,3,
$$

где индекс $\alpha$ нумерует всевозможные двухчастичные подсистемы, связанные друг с другом ортогональными преобразованиями

$$
\left(\begin{array}{c}
k_{\alpha} \\
p_{\alpha}
\end{array}\right)=U_{\alpha \beta}\left(\begin{array}{c}
k_{\beta} \\
p_{\beta}
\end{array}\right), \quad U_{\alpha \beta}=\left(\begin{array}{cc}
c_{\alpha \beta} & s_{\alpha \beta} \\
s_{\alpha \beta} & -c_{\alpha \beta}
\end{array}\right) .
$$

Матрицы $U_{\alpha \beta}$ связаны условием

$$
U_{\alpha \beta} U_{\beta \gamma} U_{\gamma \delta}=1
$$

Коэффициенты $c_{\alpha \beta}, s_{\alpha \beta}\left(c_{\alpha \beta}^{2}+s_{\alpha \beta}^{2}=1\right)$ явно выражаются через массы частиц [17], так что свободный гамильтониан $H$ в пространстве $L_{2}\left(\mathbb{R}^{9}\right)$

$$
H=-\frac{1}{2 m_{1}} \Delta_{x_{1}}-\frac{1}{2 m_{2}} \Delta_{x_{2}}-\frac{1}{2 m_{3}} \Delta_{x_{3}}
$$

где $x_{1}, x_{2}, x_{3}$ - координаты частиц в конфигурационном пространстве после отделения движения центра масс, унитарно эквивалентен оператору умножения на $P^{2}, P \in \mathbb{R}^{6}$, в пространстве

$$
\mathcal{H}^{\mathrm{ex}}=L_{2}\left(\mathbb{R}^{6}\right)
$$

С каждой двухчастичной подсистемой $\alpha \quad(\alpha=1,2,3)$ свяжем некоторое гильбертово пространство (внутренних степеней свободы) $\mathcal{H}_{\alpha}^{\text {in }}$, самосопряженный оператор $A_{\alpha}$ (внутренний гамильтониан, действующий в пространстве $\mathcal{H}_{\alpha}^{\text {in }}$ ) и некоторьй элемент $g_{\alpha} \in \mathcal{H}_{\alpha}^{\text {in }}$ (каналовый вектор). Кроме того, будем считать, что задан автоморфизм верхней полуплоскости

$$
z \rightarrow \frac{a_{\alpha}+b_{\alpha} z}{c_{\alpha}+d_{\alpha} z}
$$


так что

$$
\operatorname{det}\left(\begin{array}{cc}
a_{\alpha} & b_{\alpha} \\
\bar{c}_{\alpha} & \bar{d}_{\alpha}
\end{array}\right)=-1,
$$

а комплексные числа $a_{\alpha}, b_{\alpha}, c_{\alpha}, d_{\alpha}$ лежат на одной прямой $\left\{z=x+i y: x=\theta_{\alpha} y\right\}$ в комплексной плоскости $\mathbb{C}, \theta_{\alpha} \in \mathbb{R}$ фиксировано.

Указанные ингредиенты определяют модель $\delta$-взаимодействия с внутренней структурой, предложенную Павловым [15]. Мы следуем обозначениям, принятым в работе [13], где можно найти описание оператора энергии $h_{\alpha}$ системы двух частиц как самосопряженного оператора в пространстве $L_{2}\left(\mathbb{R}^{3}\right) \oplus \mathcal{H}_{\alpha}^{\text {in }}$.

Отметим наиболее характерные особенности модели, отсылая за подробностями к [12-14]. Резольвента оператора $h_{\alpha}$ имеет блочную структуру, и ее блок, отвечающий пространству $L_{2}\left(\mathbb{R}^{3}\right)$ (обобшенная резольвента Крейна) представляется в виде

$$
\left.P_{L_{2}\left(\mathbb{R}^{3}\right)}\left(h_{\alpha}-z\right)^{-1}\right|_{L_{2}\left(\mathbb{R}^{3}\right)}=(\hat{h}-z)^{-1}-T(z)
$$

Здесь $P_{L_{2}\left(\mathbb{R}^{3}\right)}$ - ортогональньй проектор из пространства $L_{2}\left(\mathbb{R}^{3}\right) \oplus \mathcal{H}_{\alpha}^{\text {in }}$ на подпространство $L_{2}\left(\mathbb{R}^{3}\right), \hat{h}$ - оператор умножения на $p^{2}$ в пространстве $L_{2}\left(\mathbb{R}^{3}\right)$ (свободный гамильтониан) и, наконец, $T(z)$ - одномерный интегральный оператор, имеющий ядро

$$
T(p, k ; z)=\frac{1}{p^{2}-z} t_{\alpha}(z) \frac{1}{k^{2}-z} .
$$

Функция $t_{\alpha}(z)$, которую мы в дальнейшем, допуская вольность речи, будем называть $t$-матрицей, отвечающей двухчастичной подсистеме $\alpha$, представляется в виде

$$
t_{\alpha}(z)=\frac{1}{\omega_{\alpha}(z)+\frac{i}{\sqrt{z} 4 \pi}},
$$

где $\omega_{\alpha}$ является функцией с положительной мнимой частью в верхней полуплоскости $(R$-функцией) и выражается с точностью до константы

$$
\omega_{\alpha}(z)=\frac{d_{\alpha}+b_{\alpha} r(z)}{c_{\alpha}+a_{\alpha} r(z)}+\text { const }
$$

через дробно-линейное преобразование квадратичной формы резольвенты (вычисленной на каналовом векторе $g_{\alpha}$ ) внутреннего оператора $A_{\alpha}$ :

$$
r(z)=\left\langle\left(A_{\alpha}-z\right)^{-1} g_{\alpha}, g_{\alpha}\right\rangle .
$$

В зависимости от того, равен ли параметр модели $c_{\alpha}$ в $(2.5)$ нулю или нет, функция $\omega_{\alpha}(z)$ имеет различное поведение при $z \rightarrow \infty$ : если $c_{\alpha}=0$, то $\omega(z)$ стремится к бесконечности при $z \rightarrow \infty$; напротив, если $c_{\alpha} \neq 0$, то функция $\omega_{\alpha}(z)$ ограничена на бесконечности. $\mathrm{B}$ связи с этим $t$-матрица системы (2.9) также имеет различное поведение:

cлучай а $\left(c_{\alpha} \neq 0\right)$ :

$$
t_{\alpha}(z)=\mathcal{O}\left(\frac{1}{|z|}\right), \quad z \rightarrow-\infty
$$


случай б $\left(c_{\alpha}=0\right)$ :

$$
t_{\alpha}(z)=\mathcal{O}\left(\frac{1}{\sqrt{|z|}}\right), \quad z \rightarrow-\infty
$$

При этом формально определенная $S$-матрица модели

$$
S_{\alpha}(k)=\frac{\omega_{\alpha}\left(k^{2}\right)-\frac{i k}{4 \pi}}{\omega_{\alpha}\left(k^{2}\right)+\frac{i k}{4 \pi}}
$$

в пределе $k \rightarrow \infty$ стремится в случае "а" к единице (1.4), а в случае "б" обладает "аномальным" поведением (1.5).

Перейдем к описанию трехчастичного оператора энергии с парными взаимодействиями, которые задают двухчастичные операторы $h_{\alpha}, \alpha=1,2,3$.

Каждая система координат $\left\{p_{\alpha}, k_{\alpha}\right\}$ определяет в пространстве $\mathbb{R}^{6}$ трехмерную плоскость $\mathcal{M}_{\alpha}$ :

$$
\mathcal{M}_{\alpha}=\left\{P: k_{\alpha}=0\right\}, \quad \alpha=1,2,3 .
$$

Трехчастичный предгамильтониан $H$ системы трех частиц с внутренней структурой действует в гильбертовом пространстве (четырехкомпонентных функций)

$$
\mathcal{H}=\mathcal{H}^{\text {ex }} \oplus \mathcal{H}^{\text {in }}
$$

где

$$
\mathcal{H}^{\text {in }}=\oplus_{\alpha}\left\{L_{2}\left(\mathcal{M}_{\alpha}\right) \otimes \mathcal{H}_{\alpha}^{\text {in }}\right\}
$$

Пусть $L_{2}^{\delta}\left(\mathbb{R}^{n}\right)$ обозначает гильбертово пространство функций $f$, таких что

$$
\|f\|_{\delta}^{2}=\int_{\mathbb{R}^{n}}\left(1+P^{2}\right)^{\delta}|f(P)|^{2} d P<\infty
$$

В пространстве $\mathcal{H}^{\mathrm{ex}}=L_{2}\left(\mathbb{R}^{6}\right)$ рассмотрим область

$$
\mathcal{D}=L_{2}^{2}\left(\mathbb{R}^{6}\right)+\mathcal{N}
$$

где

$$
\mathcal{N}=\left\{\Phi \in L_{2}\left(\mathbb{R}^{6}\right): \Phi(P)=\sum_{\alpha} \frac{\varphi_{\alpha}\left(p_{\alpha}\right)}{P^{2}+1}, \quad \varphi_{\alpha} \in L_{2}^{2}\left(\mathcal{M}_{\alpha}\right)\right\}
$$

Заметим, что всякий элемент $u^{\mathrm{ex}} \in \mathcal{D}$ имеет единственное представление в виде

$$
u^{\mathrm{ex}}(P)=u(P)+\Phi(P)
$$

где

$$
\Phi(P)=\sum_{\alpha} \frac{\varphi_{\alpha}\left(p_{\alpha}\right)}{P^{2}+1},
$$

причем “плотности" $\varphi_{\alpha}$ являются элементами пространства $L_{2}^{2}\left(\mathcal{M}_{\alpha}\right)$ и

$$
u \in L_{2}^{2}\left(\mathbb{R}^{6}\right) .
$$


В координатном представлении пространство $\mathcal{N}$ состоит из функций, представимых в виде потенциалов простых слоев с плотностями $\hat{\varphi}_{\alpha}$ из классов Соболева $W_{2}^{2}\left(\mathbb{R}^{3}\right)$, "размазанных" по плоскостям $x_{i}=x_{j}$, где взаимодействуют частицы пары $\alpha=\{i j\}$.

На области

$$
\mathcal{D}\left(H_{0}\right)=\mathcal{D} \oplus_{\alpha}\left\{L_{2}^{2}\left(\mathcal{M}_{\alpha}\right) \otimes \mathcal{H}_{\alpha}^{\text {in }}\right\}
$$

рассмотрим оператор $H_{0}$, действующий по правилу

$$
H_{0}:\left(\begin{array}{c}
u+\Phi \\
\underset{\alpha}{\oplus} u_{\alpha}
\end{array}\right) \longrightarrow\left(\begin{array}{c}
P^{2} u-\Phi \\
\underset{\alpha}{\oplus}\left\{\left(A_{\alpha}+p_{\alpha}^{2}\right) u_{\alpha}+l_{\alpha} g_{\alpha}\right\}
\end{array}\right) .
$$

Здесь

$$
l_{\alpha}\left(p_{\alpha}\right)=a_{\alpha} I_{\alpha}\left(u^{\mathrm{ex}}\right)+b_{\alpha} F_{\alpha}\left(u^{\mathrm{ex}}\right),
$$

где $I_{\alpha}$ и $F_{\alpha}$-неограниченные операторы вложения пространства $L_{2}\left(\mathbb{R}^{3}\right)$ в пространство $L_{2}\left(\mathcal{M}_{\alpha}\right)$ :

$$
\begin{gathered}
I_{\alpha}\left(u^{\mathrm{ex}}\right)\left(p_{\alpha}\right)=\int_{\mathcal{M}_{\alpha}^{\perp}} d k_{\alpha}\left(u^{\mathrm{ex}}(P)-\frac{\varphi_{\alpha}\left(p_{\alpha}\right)}{P^{2}+1}\right)+2 \pi\left(\sqrt{1+p_{\alpha}^{2}}-1\right) \phi_{\alpha}\left(p_{\alpha}\right), \\
F_{\alpha}\left(u^{\mathrm{ex}}\right)\left(p_{\alpha}\right)=\varphi\left(p_{\alpha}\right) .
\end{gathered}
$$

Трехчастичный предгамильтониан $H$ определим как сужение оператора $H_{0}$ на множество $\mathcal{D}(H)$ функций из $\mathcal{D}\left(H_{0}\right)$, которое удовлетворяет связям - "граничным условиям":

$$
\left\langle u_{\alpha}, g_{\alpha}\right\rangle_{\mathcal{H}_{\alpha}^{\text {in }}}\left(p_{\alpha}\right)=c_{\alpha}\left(I_{\alpha}\left(u^{\mathrm{ex}}\right)\right)\left(p_{\alpha}\right)+d_{\alpha}\left(F_{\alpha}\left(u^{\mathrm{ex}}\right)\right)\left(p_{\alpha}\right), \quad \alpha=1,2,3
$$

Используя соотношения (2.29), можно проверить, что оператор $H$ на области $\mathcal{D}(H)$ плотно задан и симметричен.

Отметим, что если в описанной конструкции формально положить $g_{\alpha}=0$, то подпространство $\mathcal{H}^{\text {ex }}=L_{2}^{2}\left(\mathbb{R}^{6}\right)$ окажется инвариантным для оператора $H$, а граничные условия (2.29) при $g_{\alpha}=0$ перейдут в так называемые граничные условия Скорнякова-Тер-Мартиросяна, которые связывают значения функций $I_{\alpha}\left(u^{\mathrm{ex}}\right)$ и $F_{\alpha}\left(u^{\mathrm{ex}}\right)$ на плоскостях $\mathcal{M}_{\alpha}$. Получившийся в результате такого формального перехода гамильтониан есть не что иное, как предгамильтониан модели Фаддеева-Минлоса $[1,2]$, Мельникова-Минлоса [9], который описывает систему трех частиц с обычными $\delta$-взаимодействиями.

\section{3. ВСПОМОГАТЕЛЬНЫЙ ОПЕРАТОР $K(z)$}

Анализ условий существенной самосопряженности предгамильтониана $H(2.25)$ на области $\mathcal{D}(H)(2.24),(2.29)$ тесно связан с изучением вспомогательного оператора $K(z)$, через который выражается трехчастичная $T$-матрица. Прежде чем определить этот объект, напомним, что $t$-матрицы в моделях точечных взаимодействий с внутренней структурой в задаче двух частиц имеют вид

$$
t(z)=\frac{1}{\omega(z)+i \frac{\sqrt{z}}{4 \pi}}
$$


где $\omega(z)$ - некоторая $R$-функция (см. (2.10)). Если внутренний оператор $A$ в модели (2.25) является ограниченным, то в представлении Герглотца (1.3) соответствующая $R$-функции $\omega(z)$ мера $\mu$ имеет компактный носитель и поэтому при $z \rightarrow-\infty$ справедлива асимптотика

в случае а:

$$
t(z)=\frac{1}{A z+B+i \frac{\sqrt{z}}{4 \pi}}+\mathcal{O}\left(|z|^{-2}\right)
$$

в случае б:

$$
t(z)=\frac{1}{B+i \frac{\sqrt{z}}{4 \pi}}+\mathcal{O}\left(|z|^{-\frac{3}{2}}\right)
$$

где $A$ и $B(A>0)$ - некоторые константы. Следовательно, при достаточно больших отрицательных значениях параметра $z$ функция $t(z)$ отделена от нуля и отрицательна. Заметим, что главный член асимптотики $t(z)$ в случае "а" совпадает с $t$-матрицей модели Шондина [10], а в случае "б” отвечает $t$-матрице обычного $\delta$-взаимодействия [18].

Для каждого $\alpha(\alpha=1,2,3)$ в пространстве $L_{2}\left(\mathbb{R}^{3}\right)$ рассмотрим самосопряженный строго положительный (неограниченньй) оператор умножения $W_{\alpha}(z)$ :

$$
\left(W_{\alpha}(z) f\right)(p)=\left(-t_{\alpha}\left(z-p^{2}\right)\right)^{-1} f(p),
$$

заданный на своей естественной области определения, где он самосопряжен:

$$
\mathcal{D}\left(W_{\alpha}(z)\right)=\left\{f \in L_{2}\left(\mathbb{R}^{3}\right): W_{\alpha}(z) f \in L_{2}\left(\mathbb{R}^{3}\right)\right\} .
$$

При достаточно больших по модулю отрицательных значениях параметра $z$ оператор $W_{\alpha}(z)$ в случае "а" сравним с оператором умножения на функцию $p^{2}+1$, а в случае "б" - с оператором умножения на функцию $\sqrt{p^{2}+1}$, поэтому

в случае а:

$$
\mathcal{D}\left(W_{\alpha}(z)\right)=L_{2}^{2}\left(\mathbb{R}^{3}\right),
$$

в случае б:

$$
\mathcal{D}\left(W_{\alpha}(z)\right)=L_{2}^{1}\left(\mathbb{R}^{3}\right) .
$$

Вспомогательный оператор $K(z)$ действует в пространстве $\mathcal{H}$ трехкомпонентных функций

$$
\mathcal{H}=L_{2}\left(\mathbb{R}^{3}\right) \oplus L_{2}\left(\mathbb{R}^{3}\right) \oplus L_{2}\left(\mathbb{R}^{3}\right)
$$

и является возмущением

$$
K(z)=W(z)-R(z)
$$

диагонального оператора $W(z)$

$$
W(z)=\operatorname{diag}\left(W_{1}(z), W_{2}(z), W_{3}(z)\right),
$$

где возмушение $R(z)$ представляет собой $3 \times 3$-операторную матрицу с нулевой диагональю. Матричные элементы $R(z)$ суть (неограниченные) интегральные операторы, заданные ядрами

$$
R_{\alpha \beta}(p, k ; z)=\frac{1}{\left|s_{\alpha \beta}\right|}\left(p^{2}-2 c_{\alpha \beta}(p, k)+k^{2}-z s_{\alpha \beta}^{2}\right)^{-1},
$$


где $s_{\alpha \beta}, c_{\alpha \beta}$ - элементы матрищы перехода (2.2) от одной системы координат Якоби к другой.

Время от времени, когда зависимость от параметра $z$ рассматриваемых объектов представляется нам несущественной, мы будем опускать соответствующую параметризацию в обозначениях. Оператор $R$ будем рассматривать на области

$$
\mathcal{D}(R)=\mathcal{H}^{2,2,2},
$$

где он очевидным образом корректно определен и симметричен. Здесь через $\mathcal{H}^{a, b, c}$ обозначено пространство

$$
\mathcal{H}^{a, b, c}=L_{2}^{a}\left(\mathbb{R}^{3}\right) \oplus L_{2}^{b}\left(\mathbb{R}^{3}\right) \oplus L_{2}^{c}\left(\mathbb{R}^{3}\right) .
$$

Оператор $K(z)$ будем считать заданным на области

$$
\mathcal{D}(K(z))=\mathcal{D}(W(z)) \cap \mathcal{D}(R)=\mathcal{H}^{2,2,2} .
$$

Как мы уже отмечали, имеются четыре типа трехчастичных систем с внутренней структурой:

случай I: в каждой из подсистем реализуется случай “а";

случай II: только в одной из подсистем реализуется случай "б";

случай III: только в одной из подсистем реализуется случай "a";

случай IV: в каждой из подсистем реализуется случай "б”.

Следующие два утверждения являются ключевыми как при описании самосопряженных расширений предгамильтониана $H(2.25),(2.26)-(2.29)$, заданного первоначально на $\mathcal{D}(H)$, так и в вопросах описания местоположения дискретного спектра таких расширений.

ЛЕмма 3.1. Вспомогательный оператор $K(z)$ при некотором значении $z \in \mathbb{R}_{-}$ в существенном самосопряжен на области $\mathcal{H}^{2,2,2}$ тогда и только тогда, когда трехчастичный предгамильтониан $H$ в существенном самосопряжен на $\mathcal{D}(H)$.

Заметим, что факт существенной самосопряженности $K(z)$ не зависит от выбора конкретного значения параметра $z$ (лежащего левее некоторого фиксированного $z_{0}$ ), поскольку разность $K(z)-K\left(z^{\prime}\right)$ является ограниченным оператором, точнее, продолжается до ограниченного.

Каждому самосопряженному расширению оператора $K(z)$ можно однозначно сопоставить самосопряженное расширение предгамильтониана $H$. Это соответствие можно установить так, чтобы выполнялось следующее свойство: пусть $\hat{K}(z)$ - некоторое самосопряженное расширение оператора $K(z)$ и $\hat{H}$ - соответствуюшее самосопряженное расширение оператора $H$ и пусть $\hat{K}\left(z^{\prime}\right)$ - некоторое самосопряженное расширение оператора $K\left(z^{\prime}\right)$, отвечаюшего другой точке $z^{\prime}$, и $\widetilde{H}$ - соответствуюшее самосопряженное расширение оператора $H$. Тогда $\hat{H}=\widetilde{H}$ тогда и только тогда, когда $\mathcal{D}(\hat{K}(z))=\mathcal{D}\left(\hat{K}\left(z^{\prime}\right)\right)$.

Лемма 3.2. Пусть $\widetilde{K}(z)$ - некоторое самосопряженное расширение оператора $K(z)$, а $\widetilde{H}$ - соответствующее самосопряжсенное расширение предгамильтониа на $H$. Тогда вещественная точка z принадлежит резольвентному множеству оператора $\widetilde{H}$ тогда и только тогда, когда оператор $\widetilde{K}(z)$ ограниченно обратим.

Доказательства этих утверждений вполне стандартны и, по существу, ничем не отличаются от рассуждений, предложенных в $[2,9,19]$. 
Таким образом, теория самосопряженных расширений предгамильтониана $H$ сводится к изучению самосопряженных расширений оператора $K(z)$, а доказательство полуограниченности оператора энергии - к вопросам обратимости $K(z)$, точнее его самосопряженных расширений, при достаточно больших по модулю отрицательных значениях параметра $z$.

Ключевой в техническом отношении для нас является следующая

Лемма 3.3. Рассмотрим в пространстве $L_{2}\left(\mathbb{R}^{3}\right)$ интегральный оператор $Q$ с ядром

$$
Q(p, k)=\frac{1}{\left(p^{2}+1\right)^{\frac{1}{4}}\left(p^{2}+k^{2}+1\right)\left(k^{2}+1\right)^{\frac{1}{4}}} .
$$

Этот оператор ограничен в пространстве $L_{2}\left(\mathbb{R}^{3}\right)$. Более того, $Q$ непрерывно отображает пространство $L_{2}^{\delta}\left(\mathbb{R}^{3}\right), \quad 0 \leq \delta<1$, в себя, и, следовательно, оператор $Q$ непреръвно отображсает пространство $L_{2}^{\delta}\left(\mathbb{R}^{3}\right), \delta \geq 1$, в пространство $L_{2}^{\kappa}\left(\mathbb{R}^{3}\right)$ при любом $\kappa<1$.

ДокАЗАТЕЛьСтво. Доказательство ограниченности оператора $Q$ в пространстве $L_{2}\left(\mathbb{R}^{3}\right)$ можно найти в работе [6]. Для доказательства его ограниченности в пространстве $L_{2}^{\delta}\left(\mathbb{R}^{3}\right), 0<\delta<1$, нам потребуется минимальное усовершенствование предложенной в [6] оценки. Пусть $\delta<1$. Выберем такое $\varepsilon>0$, что $\delta+\varepsilon<1$. Пусть $f \in L_{2}^{\delta}\left(\mathbb{R}^{3}\right)$ и $q=Q f$. Применяя неравенство Коши-Буняковского, имеем неравенство

$$
|q(p)|^{2} \leq \frac{1}{\sqrt{p^{2}+1}} \int_{\mathbb{R}^{3}} \frac{d k}{\left(p^{2}+k^{2}+1\right)\left(k^{2}+1\right)^{\frac{1}{2}+\delta+\varepsilon}} \int_{\mathbb{R}^{3}} \frac{d k|f(k)|^{2}\left(k^{2}+1\right)^{\delta+\varepsilon}}{\left(p^{2}+k^{2}+1\right)}
$$

Для интеграла

$$
I(p)=\int_{\mathbb{R}^{3}} \frac{d k}{\left(p^{2}+k^{2}+1\right)\left(k^{2}+1\right)^{\frac{1}{2}+\delta+\varepsilon}}
$$

справедлива оценка

$$
I(p) \leq \int_{\mathbb{R}^{3}} \frac{d k}{\left(p^{2}+k^{2}+1\right)|k|^{1+2 \delta+2 \varepsilon}} \leq \operatorname{const}\left(p^{2}+1\right)^{-(\delta+\varepsilon)}
$$

а следовательно,

$$
\begin{aligned}
\|q\|_{L_{2}^{\delta}}^{2} & \leq \int_{\mathbb{R}^{3}} \frac{d p}{\left(p^{2}+1\right)^{\varepsilon+\frac{1}{2}}} \int_{\mathbb{R}^{3}} \frac{d k|f(k)|^{2}\left(k^{2}+1\right)^{\delta+\varepsilon}}{\left(p^{2}+k^{2}+1\right)}= \\
& =\int_{\mathbb{R}^{3}} d k|f(k)|^{2}\left(k^{2}+1\right)^{\delta+\varepsilon} \int_{\mathbb{R}^{3}} \frac{d p}{\left(p^{2}+1\right)^{\varepsilon+\frac{1}{2}}\left(p^{2}+k^{2}+1\right)} \leq\|f\|_{L_{2}^{\delta}}^{2}
\end{aligned}
$$

Заметим, что введение "в игру" параметра $\varepsilon$ обеспечивает сходимость последнего из интегралов в оценке (3.17). Это неравенство доказывает первое утверждение леммы.

Доказательство оставшегося утверждения основано на том, что любое пространство “быстроубывающих функций" $L_{2}^{\delta}\left(\mathbb{R}^{3}\right)$ при $\delta \geq 1$ содержится в пространстве $L_{2}^{\kappa}\left(\mathbb{R}^{3}\right)$ при $\kappa<1$, которое оператор $Q$ отображает в себя. 
На основании леммы 3.3. мы можем сделать два важных наблюдения. Первое состоит в том, что оператор $R$, заданный на $\mathcal{D}(R)=\mathcal{H}^{2,2,2}$, допускает симметричное расширение $\hat{R}$ как интегральный оператор с ядром (3.10) на более широкой области

$$
\mathcal{D}(\hat{R})=\mathcal{H}^{1,1,1} .
$$

Для доказательства заметим, что ядра интегральных операторов $R_{\alpha \beta}(z)$ допускают оценки

$$
\left|R_{\alpha \beta}(k, p ; z)\right| \leq \frac{\text { const }}{p^{2}+k^{2}+1}
$$

и что $R_{\alpha \beta}(z) f \in L_{2}\left(\mathbb{R}^{3}\right)$ при $f \in L_{2}^{1}\left(\mathbb{R}^{3}\right)$ в силу непрерывности оператора $Q$ из леммы 3.3 в пространстве $L_{2}^{\frac{1}{2}}$. Таким образом, в пространстве $\mathcal{H}$ на области $\mathcal{D}(\hat{R})$ корректно определен оператор $\hat{R}$. Его симметричность на этой области проверяется непосредственно.

Область определения оператора $W$ в силу (3.6), (3.7) совпадает с $\mathcal{H}^{2,2,2}$ лишь в случае I, в остальных случаях она несколько шире. Так, например, в случае IV

$$
\mathcal{D}(W)=\mathcal{H}^{1,1,1},
$$

при этом тем не менее в каждом из случаев I-IV

$$
\mathcal{D}(W) \subset \mathcal{D}(\hat{R})=\mathcal{H}^{1,1,1} .
$$

Второе наблюдение состоит в том, что оператор $K$, рассматриваемьй как возмушение оператора $W$,

$$
\hat{K}=W-\hat{R},
$$

определен не только на области $\mathcal{H}^{2,2,2}$, но и на более широкой (в случаях II - IV) области $\mathcal{D}(W)$

$$
\mathcal{D}(\hat{K})=\mathcal{D}(W) \cap \mathcal{D}(\hat{R})=\mathcal{D}(W) .
$$

Мы запишем оператор $\hat{K}(z)$ в несколько другом, симметризованном виде. Для этого введем операторы

$$
B_{\alpha \beta}(z)=W_{\alpha}^{-\frac{1}{2}}(z) R_{\alpha \beta}(z) W_{\beta}^{-\frac{1}{2}}(z),
$$

заданные первоначально на классе быстроубывающих функций. Операторы $B_{\alpha \beta}$ являются интегральными операторами с ядрами

$$
B_{\alpha \beta}(p, k ; z)=\left(-t_{\alpha}\left(z-p^{2}\right)\right)^{\frac{1}{2}} R_{\alpha \beta}(p, k ; z)\left(-t_{\beta}\left(z-k^{2}\right)\right)^{\frac{1}{2}},
$$

которые допускают оценку

$$
\left|B_{\alpha \beta}(p, k ; z)\right| \leq \frac{\text { const }}{\left(p^{2}+1\right)^{\delta_{\alpha}}\left(p^{2}+k^{2}+1\right)\left(k^{2}+1\right)^{\delta_{\beta}}},
$$

где $\delta_{\alpha}=\frac{1}{2}$, если в двухчастичной подсистеме $\alpha t$-матрица $t_{\alpha}(z)$ удовлетворяет условию "а", и $\delta_{\alpha}=\frac{1}{4}-$ в противном случае.

В силу леммы 3.3 операторы $B_{\alpha \beta}(z)$ продолжаются до ограниченных операторов в пространстве $L_{2}\left(\mathbb{R}^{3}\right)$ и, следовательно, задают в пространстве $\mathcal{H}$ ограниченньй матричный оператор $B(z)$ с матричными элементами $B_{\alpha \beta}(z)$. При этом на области $\mathcal{D}(\hat{K}(z))$ имеет место следующее представление:

$$
\hat{K}=W-W^{\frac{1}{2}} B W^{\frac{1}{2}} .
$$

Корректность такого представления обеспечивается следующей леммой. 
ЛЕмма 3.4. Область определения оператора $W^{\frac{1}{2}}$ инвариантна относительно onepamopa $B$ :

$$
B \mathcal{D}\left(W^{\frac{1}{2}}\right) \subset \mathcal{D}\left(W^{\frac{1}{2}}\right) .
$$

ДокАЗАТЕЛЬСтво. Множество двухчастичных подсистем с точечными взаимодействиями, как мы уже отмечали, разбивается на два класса, которые мы обозначим через $\mathcal{A}$ и $\mathcal{B}$. Будем говорить, что подсистема $\alpha \in \mathcal{A}$, если соответствуюшая $t$-матрица $t_{\alpha}(z)$ удовлетворяет условию "а", и $\beta \in \mathcal{B}$, если $t_{\beta}(z)$ удовлетворяет условию "б”. Рассмотрим интегральные операторы $Q_{\alpha \beta}$, заданные ядрами

$$
Q_{\alpha \beta}(p, k ; z)=\left(-t_{\alpha}\left(z-p^{2}\right)\right)^{\theta_{\alpha}} R_{\alpha \beta}(p, k ; z)\left(-t_{\beta}\left(z-k^{2}\right)\right)^{\theta_{\beta}},
$$

где $\theta_{\alpha}=\frac{1}{4}$, если $\alpha \in \mathcal{A}$, и $\theta_{\beta}=1$, если $\beta \in \mathcal{B}$. Ядра операторов $Q_{\alpha \beta}$ очевидным образом допускают оценки

$$
\left|Q_{\alpha \beta}(p, k ; z)\right| \leq \frac{\text { const }}{\left(p^{2}+1\right)^{\frac{1}{4}}}\left(k^{2}+p^{2}+1\right)\left(k^{2}+1\right)^{\frac{1}{4}},
$$

и, следовательно, для операторов $Q_{\alpha \beta}$ справедливы все утверждения леммы 3.3 .

Заметим, что операторы $B_{\alpha \beta}$ связаны с операторами $Q_{\alpha \beta}$ следуюшими соотношениями: $B_{\alpha \beta}=W_{\alpha}^{-\frac{1}{4}} Q_{\alpha \beta} W_{\beta}^{-\frac{1}{4}}$, если $\alpha, \beta \in \mathcal{A}, B_{\alpha \beta}=W_{\alpha}^{-\frac{1}{4}} Q_{\alpha \beta}$, если $\alpha \in \mathcal{A}, \beta \in \mathcal{B}$, $B_{\alpha \beta}=Q_{\alpha \beta} W_{\beta}^{-\frac{1}{4}}$, если $\alpha \in \mathcal{B}, \beta \in \mathcal{A}$, и, наконец, $B_{\alpha \beta}=Q_{\alpha \beta}$ при $\alpha, \beta \in \mathcal{B}$.

В каждом из четырех случаев I-IV область определения оператора $W^{\frac{1}{2}}$ представима в виде

$$
\mathcal{D}\left(W^{\frac{1}{2}}\right)=L_{2}^{\delta_{\alpha}}\left(\mathbb{R}^{3}\right) \oplus L_{2}^{\delta_{\beta}}\left(\mathbb{R}^{3}\right) \oplus L_{2}^{\delta_{\gamma}}\left(\mathbb{R}^{3}\right),
$$

где $\delta_{\alpha}=1$, если $\alpha \in \mathcal{A}$, и $\delta_{\beta}=\frac{1}{2}$, если $\beta \in \mathcal{B}$. Ясно, что проверка утверждения леммы подразумевает доказательство серии включений

$$
B_{\alpha \beta} L_{2}^{\delta_{\beta}}\left(\mathbb{R}^{3}\right) \subset L_{2}^{\delta_{\alpha}}\left(\mathbb{R}^{3}\right) .
$$

В каждом из четырех случаев I-IV такая проверка основана на систематическом использовании леммы 3.3 с учетом формул, связывающих операторы $B_{\alpha \beta}$ и $Q_{\alpha \beta}$.

Приведем такое доказательство, например, для случая I. Утверждение леммы тогда сводится к проверке инвариантности пространства $L_{2}^{1}\left(\mathbb{R}^{3}\right)$ относительно операторов $B_{\alpha \beta}$.

Пусть $f \in L_{2}^{1}\left(\mathbb{R}^{3}\right)$. Тогда

$$
W_{\beta}^{-\frac{1}{4}} f \in L_{2}^{\frac{3}{2}}
$$

и, следовательно, в силу леммы 3.3

$$
Q_{\alpha \beta} W_{\beta}^{-\frac{1}{4}} f \in L_{2}^{1-\varepsilon}\left(\mathbb{R}^{3}\right)
$$

при любом сколь угодно малом $\varepsilon$. Поэтому

$$
B_{\alpha \beta} f \in L_{2}^{\frac{3}{2}}-\varepsilon \subset L_{2}^{1}\left(\mathbb{R}^{3}\right)
$$

при $\varepsilon \leq \frac{1}{2}$.

Приведем без доказательства близкий по духу результат, который можно получить используя " $\alpha \beta$ ”-комбинаторику вкупе с систематическим применением леммы 3.3 . 
Лемма 3.5. В случае I, если $f \in \mathcal{H}$, mo

$$
B f \in \mathcal{D}\left(W^{\frac{1}{2}}\right)
$$

В случае II тем же свойством обладает квадрат оператора В: если $f \in \mathcal{H}$, то

$$
B^{2} f \in \mathcal{D}\left(W^{\frac{1}{2}}\right) .
$$

СлЕДСтвИЕ 3.1. В случаях I и II всякая собственная функиия $\varphi$ оператора B, отвечающая ненулевому собственному значению $\lambda$ :

$$
B \varphi=\lambda \varphi,
$$

принадлежит области определения квадратичной форми оператора $W$ :

$$
\varphi \in \mathcal{D}\left(W^{\frac{1}{2}}\right)
$$

\section{4. СУЩЕСТВЕННАЯ САМОСОПРЯЖЕННОСТЬ ОПЕРАТОРА $K(z)$ В СЛУЧАЯХ I-II. КЛМН-теорема}

В предыдушем разделе мы описали симметричные расширения $\hat{K}$ оператора $K$ на области $\mathcal{D}(W)$, более широкой, чем первоначальная область определения $\mathcal{D}(K)=\mathcal{H}^{2,2,2}$, заданные формулой

$$
\hat{K}=W-W^{\frac{1}{2}} B W^{\frac{1}{2}} .
$$

Качественные представления о силе возмушения $R=W^{\frac{1}{2}} B W^{\frac{1}{2}}$ дает следуюшая

Tеорема 4.1. В случаях I и II оператор В компактен и принадлежит идеалу Гильберта-ШІмидта. В случаях III и IV существенный спектр оператора $B$ содержит единицу

$$
1 \in \sigma_{\mathrm{ess}}(B)
$$

вместе с некоторой ее окрестностью.

Несколько злоупотребляя терминологией, поясним, что результат теоремы 4.1 означает, что в случаях I и II возмушение $R=W^{\frac{1}{2}} B(z) W^{\frac{1}{2}}$ является $W$-компактным в смысле форм, а в случаях III и IV оно лиш $W$-ограничено в смысле форм с $W$-гранью (в смысле форм), не меньшей единицы.

Отметим, что последнее утверждение теоремы, касающееся местоположения существенного спектра оператора $B$ в случаях III и IV, - центральное место как при доказательстве существования эффекта Ефимова $[6,8]$, так и при доказательстве неограниченности оператора энергии системы трех частиц с $\delta$-взаимодействиями $[1,2,9]$.

Мы приведем лишь набросок доказательства утверждений теоремы 4.1. 
НАБРоСОК доКАЗАТЕЛЬСтВА. Ядро интегрального оператора $B_{\alpha \beta}(z)$ допускает оценку (3.23), причем в случаях I или II хотя бы один из показателей $\delta_{\alpha}$ равен $\frac{1}{2}$, и, следовательно, ядро $\left.B_{\alpha \beta}(p, k ; z)\right)$ является квадратично суммируемой функцией аргумента $P=k \oplus p$. Следовательно, оператор $B$ принадлежит идеалу Гильберта-Шмидта.

Приведем набросок доказательства второй части теоремы. Рассмотрим сперва более простой в техническом отношении случай IV. В этом случае для всех $\alpha, \alpha=1,2,3$, $t$-матрицы $t_{\alpha}(z)$ удовлетворяют условию “б” и, следовательно, мы имеем асимптотические представления

$$
t_{\alpha}(z)=\frac{1}{B_{\alpha}+i \frac{\sqrt{z}}{4 \pi}}+\mathcal{O}\left(|z|^{-\frac{3}{2}}\right), \quad \alpha=1,2,3 .
$$

Пусть $\widetilde{t}(z)-t$-матрица, отвечаюшая специальному случаю $\delta$-взаимодействия такому, что в двухчастичной системе имеется виртуальный уровень при нулевой энергии

$$
\widetilde{t}(z)=\frac{1}{i \sqrt{z} 4 \pi}
$$

Тогда в силу (4.3)

$$
t_{\alpha}(z)=\widetilde{t}(z)+\mathcal{O}\left(\frac{1}{|z|}\right)
$$

Введем операторы $W_{\alpha}(z)$ (одинаковые при всех $\alpha$ )

$$
\widetilde{W}_{\alpha}(z)=\left(-\widetilde{t}\left(z-p^{2}\right)\right)^{-1}
$$

и операторы $\widetilde{B}_{\alpha \beta}(z)$, заданные формулой

$$
\widetilde{B}_{\alpha \beta}(z)=W_{\alpha}^{\frac{1}{2}} R_{\alpha \beta}(z) W_{\beta}^{\frac{1}{2}}
$$

Как показывает прямая оценка с учетом асимптотики (4.5), ядро разности интегральных операторов $B_{\alpha \beta}(z)-\widetilde{B}_{\alpha \beta}(z)$ является ядром Гильберта-Шмидта, и поэтому опеpaтор $\widetilde{B}_{\alpha \beta}(z)$ - компактное возмущение оператора $B_{\alpha \beta}(z)$. Пусть $\mathcal{B}$ - единичный шар в пространстве $\mathbb{R}^{3}$ и $\mathcal{P}$ - ортогональный проектор из пространства $L_{2}\left(\mathbb{R}^{3}\right)=L_{2}(\mathcal{B}) \oplus$ $L_{2}\left(\mathbb{R}^{3} \backslash \mathcal{B}\right)$ на подпространство $L_{2}\left(\mathbb{R}^{3} \backslash \mathcal{B}\right)$. Аналогичная оценка ядра

$$
\frac{1}{\left(-z+p^{2}\right)^{\frac{1}{4}}} R_{\alpha \beta}(p, k ; z) \frac{1-\theta(p)}{\left(-z+k^{2}\right)^{\frac{1}{4}}}-\frac{1-\theta(k)}{\sqrt{p}} R_{\alpha \beta} \frac{1}{\sqrt{k}}
$$

где $\theta(p)$ - индикатор единичного шара $\mathcal{B}$ в $\mathbb{R}^{3}$, показывает, что разность $\widetilde{B}(z)-\mathcal{P} \widetilde{B}(0) \mathcal{P}$ также является оператором Гильберта-Шмидта. Следовательно, в силу теоремы Вейля сушественные спектры операторов $B(z)$ и $\mathcal{P} \widetilde{B}(0) \mathcal{P}$ совпадают.

Спектральный анализ оператора $\widetilde{B}(0)$, равно как и оператора $\mathcal{P} \widetilde{B}(0) \mathcal{P}$, в известном смысле можно провести явно. Первое наблюдение состоит в том, что подпространства $L^{2}\left(\mathbb{R}^{3}\right)$, отвечаюшие фиксированному угловому моменту, являются приводяшими для операторов $\widetilde{B}_{\alpha \beta}(0)$, а второе - в том, что ядра интегральных операторов $\widetilde{B}_{\alpha \beta}$ суть однородные функции (степени $\left.-\frac{3}{2}\right)$ своих аргументов. В частности, часть оператора $\widetilde{B}_{\alpha \beta}(0)$, отвечаюшая инвариантному подпространству сфферически-симметричных 
функций, унитарно эквивалентна оператору умножения на функции (допускающие мероморфное продолжение на всю комплексную плоскость)

$$
M_{\alpha \beta}(s)=\frac{\pi}{2\left|s_{\alpha \beta}\right|} \frac{\operatorname{sh}\left(\arcsin \left|s_{\alpha \beta}\right| s\right)}{s \operatorname{ch} \frac{\pi s}{2}}
$$

в пространстве $L_{2}(\mathbb{R})$. Здесь $s_{\alpha \beta}$ - элементы матрищы перехода (2.2) от системы координат Якоби $\left(k_{\alpha}, p_{\alpha}\right)$ к системе $\left(k_{\beta}, p_{\beta}\right)$. Наиболее близкий нам по духу вывод формулы (4.9), основанньй на преобразовании Меллина, содержится в работе [9] (см. также работу [6]).

Таким образом, оператор $\widetilde{B}(0)$ имеет часть, унитарно эквивалентную оператору умножения в пространстве $L_{2}(\mathbb{R}) \oplus L_{2}(\mathbb{R}) \oplus L_{2}(\mathbb{R})$ на $3 \times 3$-матрицу-функцию $M(s)$ с нулевой диагональю, заданную согласно (4.9) матричными элементами $M_{\alpha \beta}(s), \alpha \neq \beta$. В свою очередь, оператор $\mathcal{P} \widetilde{B}(0) \mathcal{P}$ содержит часть - оператор типа Винера-Хопфа, спектр которого совпадает со спектром оператора умножения на матрицу-функцию $M(s)$ "на полуоси" [22], более точно, - в пространстве $L_{2}\left(\mathbb{R}_{+}\right) \oplus L_{2}\left(\mathbb{R}_{+}\right) \oplus L_{2}\left(\mathbb{R}_{+}\right)$. Следовательно, существенный спектр оператора $\mathcal{P} \widetilde{B}(0) \mathcal{P}$, а значит, по теореме Вейля и оператора $B(z)$ содержит множество значений, которые принимают собственные числа матришы $M(s)$ при всевозможных значениях аргумента $s \in \mathbb{R}_{+}$. Тот факт, что это множество включает в себя некоторый интервал, содержащий единицу, доказывается следующим приемом (см. [9]). Рассмотрим (непрерывную) функцию

$$
f(s)=\operatorname{det}(I-M(s))
$$

Очевидно, что

$$
\lim _{s \rightarrow \infty} f(s)=1 .
$$

Вычисляя значение детерминанта $f(s)$ в нуле, приходим к формуле

$$
f(0)=1-\prod_{(\alpha \rightarrow \beta) \in g} M_{\alpha \beta}(0)-\sum_{(\alpha \rightarrow \beta) \in g} M_{\alpha \beta}^{2}(0),
$$

где суммирование ведется по стрелкам циклического граффа $g: 1 \rightarrow 2 \rightarrow 3 \rightarrow 1$. Далее, используя элементарное неравенство

$$
\frac{2}{\pi}<\frac{\sin \varphi}{\varphi}
$$

справедливое при $0 \leq \varphi<\frac{\pi}{2}$, из (4.9) заключаем, что

$$
M_{\alpha \beta}(0)>1, \quad \alpha \neq \beta
$$

Следовательно, $f(0)<0$, а значит, в силу непрерывности $f(s)$ найдется такое $s_{0}$, что $f\left(s_{0}\right)=1$. Поскольку неравенство $f(0)<0$ строгое, это означает, что некоторая окрестность единицы содержится в существенном спектре оператора $B$.

В случае III в одной и только в одной из подсистем, скажем с индексом $\gamma$, реализуется случай "а" (т.е. соответствующая матрица рассеяния имеет "правильное" высокоэнергетическое поведение). Поэтому матричные элементы $B_{\alpha \gamma}(z)$ и $B_{\gamma \beta}(z)$ в силу 
утверж дения первой части теоремы являются операторами Гильберта-Шмидта. В результате, как и в случае IV,$B(z)$ является компактным возмущением некоторого оператора, часть которого имеет спектр такой же, как и у оператора умножения в пространстве $L_{2}\left(\mathbb{R}_{+}\right) \oplus L_{2}\left(\mathbb{R}_{+}\right) \oplus L_{2}\left(\mathbb{R}_{+}\right)$на матрицу-функцию $\widetilde{N}(s)=\left\{N_{\alpha \beta}\right\}$, имеющую всего лишь 2 отличных от нуля матричных элемента:

$$
N_{\alpha^{\star} \beta^{\star}}(s)=M_{\alpha^{\star} \beta^{\star}}(s),
$$

где $\alpha^{\star}, \beta^{\star}$ - номера двухчастичных подсистем $\left(\alpha^{\star} \neq \beta^{\star}\right)$, отличных от подсистем с номером $\gamma$.

Как и в случае IV,

$$
\lim _{s \rightarrow \infty} \operatorname{det}(I-N(s))=1
$$

$$
\operatorname{det}(I-N(0))=1-N_{\alpha^{\star} \beta^{\star}}^{2}(0)<0,
$$

что (как и в случае IV) опять же доказывает, что $1 \in \sigma_{\mathrm{ess}}(B(z))$ вместе с некоторой своей окрестностью.

Используя относительную компактность возмущения $R=W^{\frac{1}{2}} B W^{\frac{1}{2}}$ (в смысле форм) и следствие 3.1 , мы докажем, что в случаях I и II относительная $W$-грань возмушения равна нулю. Применяя КЛМН-теорему [21, теорема Х.17], мы докажем, что оператор $\hat{K}(z)$ на области $\mathcal{D}(\hat{K}(z))$ самосопряжен.

Можно показать, что в случаях III и IV $W$-грань возмушения (в смысле форм) не меньше единицы, что закрывает возможность применения КЛМН-теоремы, и, как мы покажем во второй части работы, (которая будет опубликована отдельно), оператор $\hat{K}(z)$ на $\mathcal{D}(\hat{K}(z))$ лишь симметричен, но не сушественно самосопряжен.

Tеорема 4.2. В случаях I и II оператор $\hat{K}$ на области $\mathcal{D}(\hat{K})$ самосопряжен.

ДокАЗАТЕЛЬСТво. На области $\mathcal{D}[\mathbf{k}]=\mathcal{D}\left(W^{\frac{1}{2}}\right)$ рассмотрим квадратичную форму

$$
\mathbf{k}[\varphi]=\mathbf{w}[\varphi]-\mathbf{r}[\varphi]
$$

где $\mathbf{w}-$ квадратичная форма оператора $W$ :

$$
\mathbf{w}[\varphi]=\left\langle W^{\frac{1}{2}} \varphi, W^{\frac{1}{2}} \varphi\right\rangle, \quad \varphi \in \mathcal{D}\left(W^{\frac{1}{2}}\right),
$$

а форма $\mathbf{r}$, заданная на $\mathcal{D}[\mathbf{r}]=\mathcal{D}\left(W^{\frac{1}{2}}\right)$, “отвечает" возмушению $R$ :

$$
\mathbf{r}[\varphi]=\left\langle B W^{\frac{1}{2}} \varphi, W^{\frac{1}{2}} \varphi\right\rangle .
$$

Мы покажем, что форма $\mathbf{r}$ является $\mathbf{w}$-ограниченной формой с $\mathbf{w}$-гранью нуль, т.е. для любого $\varepsilon>0$ найдется такое $b>0$, что выполнено неравенство

$$
|\mathbf{r}[\varphi]| \leq \varepsilon \mathbf{w}[\varphi]+b\|\varphi\|
$$

для всех $\varphi \in \mathcal{D}\left(W^{\frac{1}{2}}\right)$.

Первый шаг доказательства (4.19) использует компактность оператора $B$ в случаях I и II. 
Пусть $P$ - спектральный проектор оператора $B$, отвечающий компоненте спектра, лежащей в интервале $[-\varepsilon, \varepsilon]$. Тогда

$$
B=P B P+Q
$$

где $Q$ - конечномерный оператор

$$
Q \cdot=\sum_{\left|\lambda_{i}\right|>\varepsilon} \lambda_{i}\left\langle\cdot, \varphi_{i}\right\rangle \varphi_{i}
$$

Здесь через $\lambda_{i}$ и $\varphi_{i}$ обозначены собственные значения и соответствуюшие собственные функции компактного оператора $B$. Норма оператора $P B P$ не превосходит $\varepsilon$, а следовательно, справедливо неравенство

$$
|\mathbf{r}[\varphi]| \leq \varepsilon \mathbf{w}[\varphi]+\sum_{\left|\lambda_{i}\right|>\varepsilon}\left|\lambda_{i}\right|\left|\left\langle W^{\frac{1}{2}} \varphi, \varphi_{i}\right\rangle\right|^{2} .
$$

В силу следствия 3.1 второе слагаемое в правой части (4.22) является ограниченной формой, что доказывает неравенство (4.19) при

$$
b=\sum_{\left|\lambda_{i}\right|>\varepsilon}\left|\lambda_{i}\right| \cdot\left\|W^{\frac{1}{2}} \varphi_{i}\right\|^{2} .
$$

На основании КЛМН-теоремы заключаем, что форма $\mathbf{k}$ в случаях I и II замкнута и полуограничена, а следовательно, ей отвечает некоторый самосопряженный оператор. Нетрудно, однако, убедиться, что таким оператором как раз является оператор $\hat{K}$, что и завершает доказательство теоремы.

ЛЕмма 4.1. В случаях I и II трехчастичньй гамильтониан Н в существенном самосопряжен на $\mathcal{D}(H)$ и полуограничен снизу.

ДокАЗАТЕЛЬСТво. КЛМН-теорема также утверждает, что оператор $\hat{K}$ в существенном самосопряжен на любой области существенной самосопряженности невозмущенного оператора, в данном случае оператора $W$. В частности, оператор $K$ на области $\mathcal{D}(K)=\mathcal{H}^{2,2,2}$ в случаях I и II также в существенном самосопряжен (в случае I оператор $K$ самосопряжен на $\mathcal{D}(K)=\mathcal{H}^{2,2,2}$. Используя результат леммы 3.1 , заключаем, что в случаях I и II трехчастичный гамильтониан $H$ в существенном самосопряжен на $\mathcal{D}(H)$. Тем самым мы доказали первую часть утверждения леммы.

Доказательство обратимости оператора $\hat{K}(z)$ при достаточно больших по модулю отрицательных значениях параметра $z$ основано на значительно более грубом, чем при доказательстве теоремы 4.2, рассуждении: наличие суммируемой мажоранты для квадрата ядер операторов $B_{\alpha \beta}(z)$ и тот факт, что

$$
\left|B_{\alpha \beta}(p, k ; z)\right| \underset{z \rightarrow-\infty}{\rightarrow} 0
$$

при фиксированных аргументах $p$ и $k$, в силу теоремы о мажорируемой сходимости показывают, что $B(z) \rightarrow 0$ при $z \rightarrow-\infty$ в норме Гильберта-Шмидта. Следовательно, при $z$, лежаших левее некоторого $z_{0} \in \mathbb{R}_{0}$, оператор $B$ является сжатием, при этом выполнено неравенство

$$
|\mathbf{r}| \leq(1-\|B(z)\|) \mathbf{w}
$$


С другой стороны, из представления (3.2)-(3.4) следует, что нижняя грань формы w $(z)$ стремится к плюс бесконечности как $|z|$ в случае I и как $\sqrt{|z|}$ в случае II:

$$
\inf _{\|\varphi\|=1}\left\langle W^{\frac{1}{2}}(z) \varphi, W^{\frac{1}{2}} \varphi\right\rangle=\mathcal{O}\left(|z|^{\delta}\right),
$$

где $\delta=1$ в случае I и $\delta=\frac{1}{2}$ в случае II. Следовательно, при $z$, лежаших левее $z_{0}$, оператор $\hat{K}(z)$ ограниченно обратим. В силу леммы 3.2 это как раз и доказывает полуограниченность трехчастичных операторов энергии $H$ в случаях I и II.

\section{Список литературы}

[1] Р.А.Минлос, Л.Д.Фаддеев // ДАН СССР. 1961. Т. 141. №6. С. 1335-1338.

[2] Р.А.Минлос, Л.Д.Фаддеев // ЖЭТФ. 1961. Т. 41. №12. С. 1850-1851.

[3] V.Efimov // Phys. Lett. 1970. V. B33. P. 563-564.

[4] Г.В.Скорняков, К.А.Тер-Мартиросян // ЖЭТФ. 1956. Т. 31. №5. С. 775-790.

[5] Г.С.Данилов // ЖЭЭТФ. 1961. Т. 40. № 2. С. 498-507.

[6] Д.Р.Яфаев // Матем. сб. 1974. Т. 94. № 4. С. 567-593.

[7] S.Albeverio, R.Hoegh-Krohn, T.T.Wu // Phys. Lett. 1981. V. A83. P. 105-109.

[8] A.V.Sobolev // Commun. Math. Phys. 1993. V. 156. P. 127-168.

[9] A.M.Mel'nikov, R.A.Minlos // Adv. in Sov. Math. 1991. V. 5. P. 99-112.

[10] Ю.Г.Шондин // ТМФ. 1982. Т. 51. № 2. С. 181-191.

[11] L.E.Thomas // Phys. Rev. 1984. V. D30. P. 1233-1237.

[12] Б.С.Павлов // Матем. сб. 1988. Т. 136 (178). № 2. С. 163-177.

[13] К.А.Макаров // Алгебра и анализ. 1992. Т. 4. № 5. С. 155-171.

[14] К.А.Макаров, В.В.Мележик, А.К.Мотовилов. Препринт Р5-94-51. Дубна: ОИЯИ, 1994.

[15] Б.С.Павлов // ТМФ. 1984. Т. 59. № 3. С. 345-354.

[16] R.Schrader // Commun. Math. Phys. 1968. V. 10. P. 155-178.

[17] С.П.Меркурьев, Л.Д.Фаддеев. Квантовая теория рассеяния для систем нескольких частиц. М.: Наука, 1985.

[18] С.Альбеверио, Ф.Гестези, Р.Хеәг-Крон, Х.Хольден. Решаемые модели в квантовой механике. М.: Мир, 1991.

[19] S.Albeverio, K.A.Makarov. Preprint SFB-237, № 237. Bochum, 1994.

[20] P.А.Минлос, М.Х.Шерматов // Вестник МГУ, Сер. І. 1989. №6. С. 7-14.

[21] М.Рuд, С.Саймон. Методы современной математической физики. Т. 2. М.: Мир, 1978.

[22] И.Ц.Гохберг, М.Г.Крейн // УМН. 1958. Т. 13. №2(80). С. 3-72.

Отдел вычислительной и математической физики, Санкт-Петербургский Университет,

Поступила в редакцию 198094 Санкт-Петербург, Россия

Fakultät für Mathematik, Ruhr Universität Bochum, 44780 Bochum, Germany

Max Planck Arbeitsgruppe,

Potsdam University,

14415 Potsdam, Germany

\section{K. A. Makarov, V. V. Melezhik \\ THE EFIMOV EFFECT AND COLLAPS IN THREE-BODY SYSTEMS WITH POINT-LIKE INTERACTIONS. I}

The three-body systems with point like interactions with an internal structure are considered. The complete classification of these systems are carried out and the conditions when the corresponding energy operators are semi-bounded from below are studied. 